\title{
Routine screening of antenatal population for thyroid disorders-mandatory
}

\author{
Kaliki Hymavathi*, Malini Devi Gottipati, Tejaswini Jakka, Bhavana T. C.
}

\begin{abstract}
Department of Obstetrics and Gynecology, Narayana Medical College and Hospital (NMCH), Nellore, Andhra Pradesh, India
\end{abstract}

Received: 02 January 2018

Accepted: 03 February 2018

*Correspondence:

Dr. Kaliki Hymavathi,

E-mail: drhymakrreddy@yahoo.co.in

Copyright: () the author(s), publisher and licensee Medip Academy. This is an open-access article distributed under the terms of the Creative Commons Attribution Non-Commercial License, which permits unrestricted non-commercial use, distribution, and reproduction in any medium, provided the original work is properly cited.

\begin{abstract}
Background: Thyroid dysfunction is commonly seen in pregnant women causing adverse maternal and fetal effects. Routine screening of antenatal population for the same will be helpful for successful pregnancy outcomes. The objective of this study is to detect and treat antenatal women with Thyroid dysfunction to avoid deleterious maternal/ fetal effects.

Methods: The present study is a hospital based prospective study conducted in the department of Obstetrics and Gynaecology of Narayana Medical College and Hospital(NMCH), Nellore, Andhra Pradesh, India. 1000 pregnant women were randomly recruited into this study. Apart from routine antenatal investigations, Thyroid function tests (TFT) - Thyroid stimulating hormone (TSH), freeT4 (fT4) and Thyroid peroxidase antibody (TPO Ab) are done for all the recruited women at the first visit of antenatal booking and individual Thyroid tests are repeated as required.

Results: Following the TFT the study population is classified into pregnancies with euthyroidism, subclinical/ overt hypothyroidism and hyperthyroidism. The prevalence of Thyroid disorders in present study was $12.7 \%$ of which hypothyroidism noted in $10.3 \%$ (subclinical $6.9 \%$ and overt $3.4 \%$ ) while hyperthyroidism seen in $2.4 \%$ (subclinical $1.8 \%$ and overt $0.6 \%$ ). The various pregnancy complications, labour and neonatal outcomes have been analyzed.

Conclusions: Thyroid dysfunction in pregnancy affects the health of the mother as well as the baby. Screening for Thyroid dysfunction should be performed as part of the routine antenatal work-up for successful pregnancy outcomes.
\end{abstract}

Keywords: Pregnancy, Thyroid disorders, Universal screening

\section{INTRODUCTION}

Thyroid disorders have indeed become a household name these days, close behind hypertension and diabetes. The prevalence of this condition is believed to be higher in women than men. The Endocrine Society Clinical Practice Guidelines recommends that management of thyroid diseases during pregnancy requires special considerations because pregnancy induces major changes in thyroid function and maternal thyroid disease can have adverse effects on the pregnancy and the fetus. ${ }^{1}$ Maternal subclinical hypothyroidism during early pregnancy has been shown to be associated with impaired neuropsychological development of children and several other adverse outcomes, including premature birth, preeclampsia, and increased fetal mortality. ${ }^{2}$ Maternal hyperthyroidism may be accompanied by coincident problems in fetal thyroid function. Autoimmune thyroid disease is associated with increased rates of miscarriage. Disorders of Thyroid hormone production can also affect female fertility. Milder forms of both hypothyroidism and hyperthyroidism may not render a woman infertile but they may still be associated with an increased risk of miscarriage. A detailed study contemplated to understand the clinical profile including maternal and fetal complications of thyroid disorders in pregnancy would 
help the treating doctor to foresee the complications and institute timely appropriate therapy.

The aim of this study is to prove the importance of universal screening of all expectant mothers for Thyroid disorders and to spot out Thyroid dysfunction among asymptomatic antenatal mothers by introducing Thyroid screening into routine antenatal protocol so as to improve maternal and fetal outcomes.

\section{METHODS}

The present study is a hospital based prospective study conducted in the department of Obstetrics and Gynaecology of NMCH Nellore, Andhra Pradesh, India. 1000 pregnant women were randomly recruited into this study after obtaining institutional ethical committee approval and patient informed consent. Inclusion criteria being singleton pregnancy (primigravida/multigravida) and exclusion criteria being any medical disorder complicating pregnancy, multiple pregnancy, Gestational trophoblastic disease and known history of any malignancy. Inclusion and exclusion was done following detailed history (demography, complaints, period of gestation, past obstetric, medical and surgical) and clinical examination (complete general physical, systemic and obstetric). Apart from routine antenatal investigations, TFT (TSH, fT4 and TPO Ab) were done at the booking antenatal visit. Individual Thyroid tests are repeated as required during the course of pregnancy. Interventions include treatment with L-thyroxine in hypothyroid women and anti-thyroid medication in hyperthyroids (with undetectable TSH and elevated fT4). Study Measures are: prevalence of Thyroid disorders in pregnancy, adverse obstetrical and neonatal outcomes. Data collected and tabulated.

\section{RESULTS}

Following the initial TFT all the recruited women are classed as: euthyroids, subclinical/ overt hypothyroids and subclinical / overt hyperthyroids. The prevalence of
Thyroid disorders in present study (12.7\%) and both hypo /hyper shown in Table 1.

Table 1: Types of thyroid disorders-prevalence.

\begin{tabular}{|lll|}
$\begin{array}{l}\text { Type of Thyroid } \\
\text { disorder }\end{array}$ & $\mathbf{n} / \mathrm{t}$ & Percentage \\
\hline Sub clini. hypothyroids & $69 / 1000$ & 6.9 \\
\hline Overt hypothyroids & $34 / 1000$ & 3.4 \\
\hline Sub clini. hyperthyroids & $18 / 1000$ & 1.8 \\
\hline Overt hyperthyroids & $6 / 1000$ & 0.6 \\
\hline
\end{tabular}

With regard to the age distribution $44 \%$ of women were found to be in 21-25yrs of age. $58.26 \%$ of multi gravidae were found to have Thyroid dysfunction compared to $41.73 \%$ of primi gravidae.

Around $40 \%$ of women diagnosed with Thyroid dysfunction were found to have a positive family history and $50 \%$ of these women were found to be overt hyperthyroids.

Positive history of infertility was present in about $20.4 \%$ of the diagnosed cases. Major pregnancy complications observed were preeclampsia-9.7\%, preterm deliveries (PTD)- $7.7 \%$ in hypo and $8.3 \%$ in hyperthyroids. IUGR$9.7 \%$ in hypo and $8.3 \%$ in hyperthyroids.

Miscarriage rate was $6.7 \%$ in hypothyroids and $20.8 \%$ in hyper thyroids with positive TPO antibodies.

Table 2: Thyroid disorders versus maternal complications.

\begin{tabular}{|ll|}
\hline $\begin{array}{l}\text { Maternal } \\
\text { complications }\end{array}$ & Percentage \\
\hline Preeclampsia & $9.7 \%$ \\
\hline Preterm birth & $16 \%$ (hypo- $7.7 \%$ and hyper- $8.3 \%)$ \\
\hline Miscarriages & $27.5 \%$ (hypo- $6.7 \%$ and hyper- \\
& $20.8 \%$ ) \\
\hline IUGR & $18 \%$ (hypo- $9.7 \%$ and hyper- $8.3 \%)$ \\
\hline
\end{tabular}

Table 3: Thyroid disorders versus labour outcome.

\begin{tabular}{|lllll|}
\hline Type of delivery & $\begin{array}{l}\text { Subclinical hypo } \\
(\mathbf{n = 6 6 )}\end{array}$ & $\begin{array}{l}\text { Overt hypo } \\
(\mathrm{n}=30)\end{array}$ & $\begin{array}{l}\text { Subclinical hyper } \\
(\mathrm{n}=16)\end{array}$ & $\begin{array}{l}\text { Overt hyper } \\
(\mathrm{n}=3)\end{array}$ \\
\hline Vaginal deliveries & $6.06 \%$ & $10 \%$ & $18.75 \%$ & 0 \\
\hline Instrumental deliveries & $30.3 \%$ & $6.6 \%$ & $25 \%$ & $66.6 \%$ \\
\hline Elective LSCS & $18.1 \%$ & $20 \%$ & $12.5 \%$ & 0 \\
\hline Emergency LSCS & $45.4 \%$ & $63.3 \%$ & $50 \%$ & $33.3 \%$ \\
\hline
\end{tabular}

The labour outcomes in women with Thyroid dysfunctions: EM. LSCS $45.43 \%$ and $15.39 \%$ being elective CS. Instrumental vaginal delivery incidence was $12 \%$ and spontaneous vaginal deliveries $27.5 \%$. The rate of neonatal complications was $13 \%$ (Jaundice $7.8 \%$ and
RDS $3.4 \%$ ) were predominantly seen in hypothyroids. One case of delayed skeletal maturation was observed, evidenced radiologically in a baby born to a hypothyroid mother. Neonatal thyrotoxicosis was noted in a baby of hyperthyroid mother. 
Table 4: Thyroid disorders versus neonatal complications.

\begin{tabular}{|ll|}
\hline Neonatal complications & Percentage \\
\hline Neonatal jaundice & 7.8 \\
\hline Respiratory distress syndrome & 3.4 \\
\hline Delayed skeletal maturity & 0.8 \\
\hline Neonatal thyrotoxicosis & 0.8 \\
\hline
\end{tabular}

\section{DISCUSSION}

Thyroid disorders are one among the common endocrine issues in pregnant women with deleterious impact on pregnancy and the developing fetus. Hormonal changes and metabolic demands during pregnancy results in profound alteration in foeto maternal Thyroid function. However, understanding of thyroid function associated with pregnancy has evolved significantly in the past several years and new findings have yielded a new concept of gestational transient hyperthyroxinemia. A study by Haddow et al documented a relation between maternal thyroid deficiency during pregnancy and subsequent neurophysiological development of the child adversely affecting the children's subsequent performance on neuropsychological tests. Decreases in performance can occur even when the pregnant woman's hypothyroidism is mild and probably asymptomatic. The presence of high serum concentrations of antithyroid peroxidase antibodies with hypothyroidism indicates that chronic autoimmune thyroiditis as the cause of hypothyroidism. ${ }^{3}$ Thyroid abnormalities are very often subclinical in nature and not easily recognized without specific screening programs. The main diagnostic indicator of Thyroid disease is the measurement of serum TSH and free thyroxine (fT4) levels. Availability of gestation specific TSH threshold is an important aid in the accurate diagnosis and treatment of Thyroid dysfunction. ${ }^{4}$ The American association of clinical endocrinologists (AACE), American college of endocrinology and American Thyroid association (ATA) recommend that the upper limits of the normal range of TSH levels be as follows: first trimester- $2.5 \mathrm{mIU} / \mathrm{L}$; second trimester- $3.0 \mathrm{mIU} / \mathrm{L}$; third trimester- $3.5 \mathrm{mIU} / \mathrm{L} 5$. Care requires coordination among several healthcare professionals. Vaidya et al demonstrated that screening only high-risk women failed to detect $30 \%$ of hypothyroids and $69 \%$ of hyperthyroid women. ${ }^{2}$ It is now well established that not only overt but sub clinical thyroid dysfunction also has adverse maternal and fetal outcomes. So, the main aim of this prospective study is to know the prevalence of thyroid disorders in pregnancy so as to avoid their adverse effects by implementing routine antenatal Thyroid screening.

There are few data from India about the prevalence of thyroid dysfunction in pregnancy. In the study by Sahu MT et al the general prevalence of Thyroid disorders in pregnant population was found to be $11.05 \%$ whereas in present study it is $12.7 \% .^{6}$ Subclinical vs overt hypothyroidism was noticed in $6.47 \%$ and $4.58 \%$ in the study of Sahu et al whereas present corresponding figures are $6.9 \%$ and $3.4 \%$. Subclinical hypothyroidism $(\mathrm{SCH})$ with serum TSH concentration above the upper limit of the trimester-specific reference range along with normal free T4 may be associated with an adverse outcome for both the mother and offspring. ${ }^{7}$ In present study overt hypothyroids were found to be comparatively less than Subclinical hypothyroids which might be due to the implementation of present policy of universal screening for Thyroid disorders in the antenatal population so as to pick them up in the sub clinical stage itself. In the study of Hossein $\mathrm{M}$ et al prevalence of hyperthyroidism in pregnant women was $1.5 \%$ (subclinical $0.3 \%$; and overt $1.2 \%)$ and present corresponding figures are $2.4 \%(1.8 \mathrm{v} / \mathrm{s}$ $0.6 \%$ ) with more number in the subclinical than overt variety which could be again due to routine antenatal screening. ${ }^{8}$ This obviously indicates that had authors not done routine screening in present study population out of $12.7 \%$ of pregnant women with thyroid disorders $7.5 \%$ would have gone undiagnosed (excluding those with positive family history).

The mean age of pregnant women in the study of Vaidya et al was 27 yrs whereas present study showed a mean age of $24 \mathrm{yrs}$ which can be explained in terms of the Indian tradition and culture of early marriages and pregnancy. Till date no study has been done to report the Thyroid disorders in relation to parity. In present study taking parity into consideration among primigravidae: hypothyroidism (subclinical v/s overt-44.3 v/s 32.3\%) whereas hyperthyroidism (subclinical v/s overt- $50 \mathrm{v} / \mathrm{s}$ $33.3 \%$ ) while the corresponding figures for multigravidae being $55.07 \% \mathrm{v} / \mathrm{s} 67.64 \%$ and $50 \%$ v/s $66.67 \%$. With present results it is observed both hypo and hyperthyroid disorders were found to be more prevalent in multigravidae compared to primigravidae. This may be partly due to the fact that the Thyroid dysfunction in the former group must not have been spotted out during their previous pregnancies.

Positive family history is observed many a times in thyroid disorders. Vaidya et al showed that a positive family history is present in $22.8 \%$ in a study of 413 pregnant women whereas present study showed almost $40 \%$ association with family history. Studies have shown that thyroid antibodies in the first-degree relatives have been suggested to be controlled by same genes and female gender seems to be a predictive factor as regard to consistently elevated TSH levels. It looks like the same genetic factor may be operating in present population leading to a high percentage of positive family history.

Patients with either hypo or hyperthyroidism can have fertility problems although it is possible to have these diseases and still get pregnant. $\mathrm{SCH}$ is defined by an increase in serum TSH concentrations with normal free thyroxine (fT4) levels. The prevalence of SCH in sub fertile women has been reported to vary from $0.7 \%$ to $43 \% .^{9}$ History of infertility was there in $20.4 \%$ of present study population. Among them Subclinical hypo and 
hyperthyroidism was observed to an extent of $28 \%$ in each group. Hypothyroidism may be associated with ovulatory dysfunction and menstrual irregularities and once thyroxine treatment is given it restores the normal menstrual pattern and reverses the hormonal changes responsible for infertility. Treatment with L-thyroxine should be considered in women of childbearing age with $\mathrm{SCH}$ when they are planning a pregnancy. ${ }^{10}$ In present study pregnant women found to have even $\mathrm{SCH}$ were started on L-thyroxine immediately $(\mathrm{TSH} \geq 10 \mathrm{mIU} / \mathrm{L}, 1.6$ $\mu \mathrm{g} / \mathrm{kg} /$ day; $\mathrm{TSH}<10 \mathrm{mIU} / \mathrm{L}, 25-75 \mu \mathrm{g} /$ day).

Thyroid autoimmunity (TAI) is the most prevalent autoimmune condition (5\%-20\%) in women of fertile age. TAI is characterized by the presence of anti-thyroid antibodies i.e. anti-thyroperoxidase (TPOAb) and antithyroglobulin antibodies. It may remain latent, asymptomatic, or even undiagnosed for an extended period. Numerous studies have investigated the prevalence of TAI in women with subfertility. In present study out of 24 hyperthyroids 15 of them with history of infertility were found to be TPOAb positive. In a recently published meta-analysis, the presence of anti-thyroid antibodies was associated with an increased risk of unexplained subfertility.

Hypothyroidism is known to be associated with many maternal and fetal complications. The study of Leung et al and Sahu et al denotes the major pregnancy complications as preeclampsia in $17 \%$ and $12.2 \%$ respectively. present corresponding figure is $9.7 \%$. The association of hypo thyroidism and pre-eclampsia (PE) is not surprising, as hypothyroidism is an accepted cause of reversible hypertension resulting in vascular smooth muscle contraction both in systemic and renal vessels, leading to an increased diastolic pressure, peripheral vascular resistance and decreased tissue perfusion. ${ }^{12}$ Thyroid dysfunctions can be associated with proteinuria resulting in increased excretion of thyroxin and TBG that cannot be compensated by the body at times. With the known correlation between hypothyroidism and hypertension a "preeclampsia like syndrome" can occur in pregnancies complicated by hypothyroidism and the same explanation might hold good in present study also. ${ }^{13}$

Unborn babies need a supply of thyroid hormones from their mother if they are to develop properly during pregnancy. Thyroid hormones travel from the mother's bloodstream through the placenta to the baby with the help of special proteins, called transporters. A newly discovered transporter, called MCT10, seems particularly good at transporting thyroid hormones playing a key role in the supply of thyroid hormones to unborn babies. ${ }^{14}$ They think it may also affect the growth and development of the placenta. Evidence suggests abnormally low levels of MCT10 may be linked to the poor growth of unborn babies resulting in growth restriction. Researchers are testing about the importance of MCT10 in both normal pregnancies and pregnancies complicated by growth restriction. The IUGR cases observed in present study were late bookings in the second and third trimesters in association with preeclampsia. Ligand binding studies have revealed presence of high affinity nuclear receptors for T3 in placenta and human fetal brain after the 10th week of gestation. It was confirmed that T3 in human placenta stimulates production of 17-estradiol and epidermal growth factor. This suggests an important role of thyroid hormones in trophoblast development and a possibility of the alteration of this function during IUGR. ${ }^{15}$ As per the study of Kriplani17 et al IUGR incidence in thyrotoxic women was around $13 \%$ present corresponding figure being $8.33 \%$.

Next in order following preeclampsia and IUGR the major pregnancy complication in the studies of Leung11 and Sahu et al is PTD showing the incidence in the range of $7-9 \% .^{3}$ Preterm birth was almost 2-fold higher in women with $\mathrm{SCH} .{ }^{16}$ In present study the figure stands around $7.7 \%$ running close to the above range. It is not surprising that a relationship exists between PTD and hypothyroidism. The occurrence of PTD in women with hypothyroidism (subclinical/ overt) could be argued to be one further manifestation of the impact of hypothyroidism on maintenance of pregnancy to term. Critical supportive evidence of this hypothesis is provided by the randomized study by Negro et al documenting a significant decrease in PTD in euthyroid women with autoimmune thyroid disorder given Levo thyroxine. This suggests that a potential etiology of PTD was subtle hypothyroidism. The incidence of PE and PTD was significantly high in the study by Kriplani et al $(22 \% \mathrm{v} / \mathrm{s} 25 \%) .{ }^{17}$ In present study the low figures may be due to early diagnosis and treatment implications.

There are few studies in the literature with regard to miscarriage rate in pregnancy with hypothyroidism. Autoimmune thyroid disease (AITD) without overt thyroid dysfunction was significantly associated with a 3 to 5-fold increase in overall miscarriage rate. ${ }^{18}$ Pregnant women who were euthyroid in the early stages of pregnancy but with positive TPO-Abs would benefit from levo thyroxine administration to improve the outcome of pregnancy and, more specifically, reduce the rate of spontaneous miscarriage and PTD. In the study of a large group of Caucasian pregnant women $(n=984)$ levo thyroxine administration to TAI-positive women showed significant decrease in obstetrical complications, with the miscarriage rate reduced by $75 \%$ and the frequency of PTD by $69 \%$. In present study the miscarriage rate is $6.7 \%$ (7cases) in hypothyroids (subclinical / overt varieties). Specifically, SCH has been associated with miscarriages in both the first and second trimesters. In present study these women have aborted within a short time after their diagnosis and treatment. Authors feel that these hypothyroid related abortions must have occurred before the treatment has become effective. Though less in prevalence marked maternal and fetal complications were noted in pregnancies complicated by hyperthyroidism. Some studies have not classified the cases into subclinical and overt hyperthyroids. Miscarriage rate in present study 
is $20.8 \%$ which is significantly high compared to $14.5 \%$ of Negro R et al. ${ }^{19}$ Out of five hyperthyroid women, four were found to be TPO Ab positive which may be responsible for activation and reactivity of immune system against foeto placental unit and thus leading to immune imbalance resulting in miscarriage.

Labour out comes in thyroid disorders varies and may not be per se due to the disease but the resultant complications of it. Primary hypothyroidism was associated with decreased odds of spontaneous labour and increased odds of Caesarean section Study done by Krishnan $\mathrm{M}$ et al observed more cases of Caesarean sections in hypothyroidism $(62.2 \%)$ and present figure is $(60.8 \%)$ being in the par with that of the above. ${ }^{20}$ In present study more of EM.LSCS were observed both in hypo and hyperthyroid cases.

Failed inductions and uterine inertia were found to be the dominant indications. present percentage of instrumental deliveries $(12 \%)$ is found mostly in subclinical hypo and hyperthyroid women. The percentage figure for normal vaginal deliveries of Muthukrishnan $\mathbf{J}$ et al and ours are in the comparable range $(29.6 \% \mathrm{v} / \mathrm{s} 27.5 \%)$.

Still birth (SB) rate among overt hypothyroids was $2.9 \%$ in the study of Sahu M et al and study of Robert Negro et al showed the SB rate of $3.9 \%$ vs $0 \%$ in overt hypo and hyper thyroids respectively. ${ }^{21}$ Poor control of hyperthyroidism during pregnancy is also associated with increased risk of miscarriage and stillbirth. ${ }^{22}$ One still birth $(4.1 \%)$ recorded in present study found to happen in a hyperthyroid woman who was non-complaint to treatment and with irregular antenatal visits.

As per the study of C.Grigoriu et al Respiratory distress syndrome (RDS) was noticed in $10 \%$ babies of hyperthyroid women, present figure being $3.4 \%$ with a wide difference. ${ }^{23}$ Various types of neonatal complications were reported in the hypothyroid mothers. In present study neonatal jaundice is seen $7.8 \%$. One case of delayed skeletal maturation was observed, evidenced radio logically in a baby born to a hypothyroid mother. Neonatal hyperthyroidism is rare. The prevalence of Graves' disease in pregnant women is approximately $0.2 \%$.

Although it is usually stated that only $1-1.5 \%$ of their offspring will have overt hyperthyroidism this figure may be much higher. There will be one case of overt neonatal thyrotoxicosis for every 4000-50.000 deliveries. ${ }^{24}$ In present study one case of neonatal thyrotoxicosis $(0.8 \%)$ was seen in a baby of a mother with thyrotoxicosis who had her first antenatal visit in the late third trimester with a gross IUGR fetus. In this case cord blood sample collected postpartum for TFT and TSH receptor antibody estimation as neonatal thyrotoxicosis is expected to occur due to transplacental passage of TSH receptor antibody and the result was positive.

\section{CONCLUSION}

Thyroid dysfunction is one of the most common endocrine disorders of pregnancy and can affect the health of the mother as well as the baby before and after delivery. Early diagnosis and gestational programming can prevent the various complications that enter the vicious cycle of transmitting from one generation to another. Thus, routine screening of the antenatal women for thyroid disorders should be made mandatory. In fact, it is better to screen and treat women in their pre-pregnant period itself to make sure of their euthyroidism creating awareness about the importance of thyroid gland through various measures aiming to make every expectant mother free of thyroid diseases.

Funding: No funding sources

Conflict of interest: None declared

Ethical approval: The study was approved by the Institutional Ethics Committee

\section{REFERENCES}

1. Endocrine society clinical practice guidelines-2007.

2. Vaidya B, Anthony S, Bilous M, Shields B, Drury J, Hutchison $S$ et al. Detection of Thyroid dysfunction in early pregnancy. Universal screening or high risk targeted case Finding. J Clin Endocronol Metab. 2007;92(1):203-7.

3. Haddow JE, Palomaki GE, Allan WC, Williams JR, Knight GJ, Gagnon J, et al. Maternal thyroid deficiency during pregnancy and subsequent neuropsychological development of the child. New England J Med. 1999 Aug;341(8):549-55.

4. El Baba KA, Azar ST. Thyroid dysfunction in pregnancy. Int J Gen Med. 2012;5:227.

5. Stagnaro-Green A, Abalovich M, Alexander E, Azizi F, Mestman J, Negro R, et al. Guidelines of the American Thyroid Association for the diagnosis and management of thyroid disease during pregnancy and postpartum. Thyroid. 2011;21:1081-125.

6. Sahu MT, Das V, Mittal S, Agarwal A, Sahu M. Overt and subclinical thyroid dysfunction among Indian pregnant women and its effect on maternal and fetal outcome. Arch Gynecol Obstet. 2010 Feb $1 ; 281(2): 215$.

7. Abalovich M, Amino N, Barbour LA, Cobin RH, De Groot LJ, Glinoer D, et al. Management of thyroid dysfunction during pregnancy and postpartum: An Endocrine Society Clinical Practice Guideline. The J clinical Endocrinol Metabol. 2007 Aug;92(8_supplement):s1-7.

8. Saki F, Dabbaghmanesh MH, Ghaemi SZ, Forouhari S, Omrani GR, Bakhshayeshkaram M. Thyroid function in pregnancy and its influences on maternal and fetal outcomes. Int J Endocrinol Metabol. 2014 Oct;12(4).

9. Cho MK. Thyroid dysfunction and subfertility. Clinc Exp Reprod Potential. 2015 Dec; 42(4): 131-5. 
10. Garber JR, Cobin RH, Gharib H, Hennessey JV, Klein I, Mechanick JI, et al. Clinical practice guidelines for hypothyroidism in adults: cosponsored by the American Association of Clinical Endocrinologists and the American Thyroid Association. Endocr Pract. 2012;18:988-1028.

11. Leung AS, Millar LK, Kooning PP, Montorom, Mestman J. Perinatal outcomes in hypothyroid pregnancies. Obstet Gynecol. 1993;81(3):349-53.

12. Inversetti A, Serafini A, Manzoni MF, Dolcetta Capuzzo A, Valsecchi L, Candiani M. Severe hypothyroidism causing pre-eclampsia-like syndrome. Case Reports Endocrinol. 2012;2012.

13. Stabouli S, Papakatsika S, Kotsis V. Hypothyroidism and hypertension. Expert Review Cardiovas Therapy. 2010 Nov;8(11):1559-65.

14. Chan S, Franklyn J, Loubiere L. Thyroid disorders and growth restriction in pregnancy: could a newly discovered protein hold the key to better treatment? Available at: https://www.action.org.uk/ourresearch/thyroid-disorders-and-growth-restrictionpregnancy-could-newly-discovered-protein-hold-

15. Vargova V, Macekova D, Kraus V et al. Association of thyroid gland disease with intrauterine growth retardation and fetal hypotrophy. 2013:105-15.

16. Kriplani A, Buckshee K, Bhargava VL, Takkar D, Ammini AC. Maternal and perinatal outcome in thyrotoxicosis complicating pregnancy. European $\mathrm{J}$ Obstet Gynecol Reprod Biol. 1994 May;54(3):15963.

17. Casey BM, Dashe JS, Wells CE, McIntire DD, Byrd W, Leveno KJ. Subclinical hypothyroidism and pregnancy outcomes. Obstet Gynecol. 2005 Feb;105(2):239-45.

18. Glinoer D. Miscarriage in Women with Positive Anti-TPO Antibodies: Is Thyroxine the Answer?
The J Clinical Endocrinol Metabol. 2006;91(7):2500-2.

19. Negro R, Schwartz A, Gismondi R, Tinelli A, Mangieri T, Stagnaro-Green A. Universal screening versus case finding for detection and treatment of thyroid hormonal dysfunction during pregnancy. The J Clinical Endocrinol Metabol. 2010 Apr;95(4):1699-707.

20. Muthukrishnan J, Verma A, HariKumar KVS, Ugala M. Indian J Endocrinol Metab. 2013;17(2):294-7.

21. Negro R, Schwartz A, Gismondi R, Tinelli A, Mangieri T, Stagnaro-Green A. Increased pregnancy loss rate in thyroid antibody negative women with TSH levels between 2.5 and 5.0 in the first trimester of pregnancy. The J Clinical Endocrinol Metabol. 2010 Sep;95(9):E44-8.

22. Männistö T, Mendola P, Grewal J, Xie Y, Chen Z, Laughon SK. Thyroid diseases and adverse pregnancy outcomes in a contemporary US cohort. J Clinical Endocrinol Metabol. 2013 Jul;98(7):272533.

23. Grigoriu C, Cezar C, Grigoras M, Horhoianu I, Parau C, Vîrtej P, et al. Management of hyperthyroidism in pregnancy. J Medic Life. 2008 Nov 15;1(4):390.

24. Ogilvy-Stuart AL. Neonatal thyroid disorders. Arch Dis Childhood-Fetal Neonatal Ed. 2002 Nov;87(3):F165-71.

Cite this article as: Hymavathi $\mathrm{K}$, Gottipati $\mathrm{MD}$, Jakka T, Bhavana TC. Routine screening of antenatal population for thyroid disorders- mandatory. Int $\mathrm{J}$ Reprod Contracept Obstet Gynecol 2018;7:2834-9. 